\title{
E. K. Farran and A. Karmiloff-Smith: Neurodevelopmental Disorders Across the Lifespan: A Neuroconstructivist Approach
}

\author{
Oxford University Press, Oxford, 2012, 394 pp, ISBN: 978-0-19-959481-8 (hardcover)
}

\author{
Kim Cornish
}

Published online: 17 January 2013

(C) Springer Science+Business Media New York 2013

Understanding developmental changes, both typical and atypical, as they occur throughout the lifespan is the central question that defines this book. The authors look through the lens of Williams Syndrome, a neurodevelopmental disorder that has been intensively investigated over the past decade across multiple levels of analysis (molecular, brain, cognitive, behavioural, and most recently at the environmental level). Although relatively rare, Williams syndrome provides a unique window of opportunity to explore complex yet dynamics changes in cognition as it unfolds across the lifespan. Farran and Karmiloff-Smith do an outstanding job in bringing together researchers who have made exceptional contributions to this field.

The reader will begin with an exploration of why development itself is critical for understanding neurodevelopmental disorders (known as the neuroconstructivist approach) and from the onset, this book brings to the forefront the core challenges that face researchers in achieving this goal. One of the most insightful chapters focuses on developmental trajectories and how best to capture dynamic but often subtle changes across development rather than continue to rely on a more traditional yet static approach of accruing "snapshots" of performance across different time-points. Across multiple cognitive domains that include numeracy, literacy, perception, face processing, and attention; and journeying from infancy through childhood and into adulthood, the reader is presented with compelling empirical evidence that the pattern of developmental outcomes is not necessarily the same as the pattern of low-level deficits in the start-state in infancy.
That is, one can never assume that the adult profile of cognitive and behavioural strengths and difficulties across and within domains is simply a more sophisticated version of the infant start-state.

The authors also highlight and introduce the importance of recognising the impact of 'domain-general' mechanisms from 'domain-specific' ones, and argue against the notion that you can map directly from genes to cognition. This is a difficult concept to articulate but is expertly articulated in this book; in part because real-world examples are given that clearly demonstrate the non-linearity and complex relationship between genes and developmental outcomes (see in particular the chapter by Steele et al. that focuses on cross-syndrome and cross domain development), but also because Williams syndrome is a complex neurodevelopmental disorder that involves numerous deletions of genes. Indeed, researchers for decades have been piecing together the puzzle of how specific genes map onto the Williams syndrome phenotype. The journey continues.

Overall, this is an impressive book from an internationally renowned team of researchers. It sets the scene for the next generation of developmental research to embrace a neuroconstructivist approach to investigate developmental changes across the lifespan, the constraints that shape later cognitive outcomes and the different mechanisms and pathways that lead to cross syndrome associations and dissociations. My hope is that research of the calibre described in this book will ultimately have a significant and long-lasting impact on the lives of affected children and adults, their families, clinicians and educators.
K. Cornish $(\bowtie)$

School of Psychology \& Psychiatry, Monash University,

Melbourne, Australia

e-mail: kim.cornish@monash.edu 